\title{
On the Utility of Laguerre Series for the Envelope PDF in Multipath Fading Channels
}

\begin{abstract}
Ali Abdi
Abstract - It is well known that multipath fading significantly affects the performance of communication systems. In order to incorporate the impact of this random phenomenon on system analysis and design, in many cases we need to calculate the probability density function (PDF) of the received signal envelope in multipath fading channels. In this paper, we consider a general multipath fading channel with arbitrary number of paths, where the amplitudes of multipath components are arbitrary correlated positive random variables, independent of phases, whereas the phases are independent and identically distributed random variables with uniform distributions. Since the integral form of the envelope PDF for such a general channel model is too complicated to be used for analytic calculations, we propose two infinite expansions for the PDF, a Laguerre series and a power series. Based on the tight uniform upper bounds on the truncation error of these two infinite series, we show that the Laguerre series is superior to the power series due to the fact that for a fixed number of terms, it yields a smaller truncation error. This Laguerre series with a finite number of terms, which expresses the envelope PDF just in terms of simple polynomial-exponential kernels, is particularly useful for mathematical performance prediction of communication systems in those indoor and outdoor multipath propagation environments, where the number of strong multipath components is small.
\end{abstract}

Index Terms-Fading channels, Wireless channels, Multipath propagation, Rayleigh fading, Radar clutter, Light scattering, Envelope distribution, Random vectors, Sum of sinusoids, Infinite expansions, Laguerre polynomials, Truncation error, Performance analysis, Bit error rate.

Contact Author: Ali Abdi (Associate Professor)

Dept. of Electrical and Computer Engineering

New Jersey Institute of Technology

323 King Blvd.

Newark, NJ 07102, USA

Phone: (973) 5965621

Fax: (973) 5965680

Email: ali.abdi@njit.edu 


\section{INTRODUCTION}

In a variety of situations encountered in communication engineering, the multipath structure of the transmission channel is such that the received signal exhibits strong fluctuations. These fluctuations are generally referred to as multipath fading, and over the past few decades, a great deal of attention has been focused on its characterization.

In this paper, we study the envelope probability density function (PDF) in detail, using a random vector model, assuming that the number of multipath components is an arbitrary constant, phases are independent with uniform distributions on $[0,2 \pi)$, while the amplitudes are dependent positive random variables with arbitrary distributions. This scenario is general enough to cover many cases of interest. For situations where the number of multipath components is a random variable or the phases have nonuniform distributions, the interested reader can refer to [1].

The rest of the paper is organized follows. The multipath fading channel model is introduced in Section II. Two infinite series are presented in Section III for the envelope PDF, a Laguerre series and a power series. Calculation of the coefficients of the series is discussed in Section IV. Upper bounds on the truncation error and coefficients of the Laguerre series are derived in Sections V and VI, respectively. The series truncation error upper bound is minimized in Section VII. By comparing the convergence rates, it is shown in Section VIII that for a fixed number of terms, the truncated Laguerre series provides a smaller truncation error than the power series. So, from this point of view, Laguerre series is preferred to the power series for calculating the envelope PDF. Application of the Laguerre series to system performance analysis in multipath channels with several dominant multipath components is briefly discussed in Section IX, as well as a numerical case study. Concluding remarks are provided in Section X.

\section{A General Model for Multipath FAding Channels}

In a multipath fading channel, multipath components can be divided into two independent groups: the first group consists of a small number of strong multipath components which do not satisfy the conditions of the central limit theorem (CLT) [2], while the second group contains a large number of weak multipath components which satisfy the CLT conditions. The first group generates a nonGaussian random process with non-Rayleigh envelope PDF, while the second group results in a Gaussian random process with Rayleigh envelope PDF. Specifically, if the signal $\vartheta_{t r}\left(t^{\prime}\right)=\cos 2 \pi f_{c} t^{\prime}$ is 
transmitted through a multipath fading channel, where $f_{c}$ is the carrier frequency and $t^{\prime}$ is the time, then the received signal has the following form:

$\vartheta_{r e}\left(t^{\prime}\right)=\vartheta\left(t^{\prime}\right)+\hat{\vartheta}\left(t^{\prime}\right)$

where $\vartheta\left(t^{\prime}\right)$ and $\hat{\vartheta}\left(t^{\prime}\right)$ are independent processes defined by:

$\vartheta\left(t^{\prime}\right)=\sum_{i=1}^{N} A_{i} \cos \left(2 \pi f_{c} t^{\prime}+\Phi_{i}\right)$,

$\hat{\vartheta}\left(t^{\prime}\right)=\sum_{i=1}^{\hat{N}} \hat{A}_{i} \cos \left(2 \pi f_{c} t^{\prime}+\hat{\Phi}_{i}\right)$.

In the above formulas, ${ }^{1} N$ and $\hat{N}$ are the number of multipath components in the first and the second groups, respectively, $A_{i}$ 's and $\hat{A}_{i}$ 's represent the amplitudes of multipath components, while $\Phi_{i}$ 's and $\hat{\Phi}_{i}$ 's stand for the phases of multipath components. In the first group $N$ is an arbitrary constant, $A_{i}$ 's are dependent positive random variables with arbitrary distributions, independent of $\Phi_{i}$ 's, and $\Phi_{i}$ 's are independent of each other with uniform distributions on $[0,2 \pi)$. A physical interpretation for these statistical properties of $\Phi_{i}$ 's is provided in [3]. The signal $\vartheta\left(t^{\prime}\right)$ generated by the first group is a nonGaussian random process with non-Rayleigh-distributed envelope. In the second group, $\hat{N}$ is large enough and the statistical properties of $\hat{A}_{i}$ 's and $\hat{\Phi}_{i}$ 's are such that based on CLT, $\hat{\vartheta}\left(t^{\prime}\right)$ can be modeled as a Gaussian random process with Rayleigh-distributed envelope. Note that since the rates of change of the number of multipath components, their amplitudes, and also their phases are much smaller than $f_{c}$, we can assume that they are (random) constants over relatively short time-intervals.

To develop the random vector model for our multipath fading channel, we note that the sums of cosine waves in (2) and (3) can be replaced by the following single cosine waves:

$\vartheta\left(t^{\prime}\right)=A \cos \left(2 \pi f_{c} t^{\prime}+\Phi\right)$

$\hat{\vartheta}\left(t^{\prime}\right)=\hat{A} \cos \left(2 \pi f_{c} t^{\prime}+\hat{\Phi}\right)$.

These representations lead to the following representation for $\vartheta_{r e}\left(t^{\prime}\right)$ in (1):

$\vartheta_{r e}\left(t^{\prime}\right)=R \cos \left(2 \pi f_{c} t^{\prime}+\Theta\right)$

${ }^{1}$ The notation in (2) and (3) is fine for narrowband transmission, where the duration of $\vartheta_{t r}\left(t^{\prime}\right)$ is large. For wideband transmission, with a short-duration $\vartheta_{t r}\left(t^{\prime}\right)$, of course $N+\hat{N}$ represents the total number of multipath components that reach the receiver with the same delay. Without loss of generality, this delay is set to zero, to simplify the notation. 
Clearly, the pairs $(A, \Phi)$ and $(\hat{A}, \hat{\Phi})$ represent the envelopes and the phases of signals from the first and the second groups of multipath components, respectively, while $R$ and $\Theta$ are the envelope and phase of the signal composed of the two groups of multipath components. The interrelationships among the random variables $A, \Phi, \hat{A}, \hat{\Phi}, R$, and $\Theta$ can be understood by using the amplitude-phase notation:

$$
A \exp (j \Phi)=\sum_{i=1}^{N} A_{i} \exp \left(j \Phi_{i}\right), \hat{A} \exp (j \hat{\Phi})=\sum_{i=1}^{\hat{N}} \hat{A}_{i} \exp \left(j \hat{\Phi}_{i}\right), \operatorname{Rexp}(j \Theta)=A \exp (j \Phi)+\hat{A} \exp (j \hat{\Phi}),
$$

where $j=\sqrt{-1}$. Similar to [4], it can be shown that the PDF's of $A$ and $R$ are given by [3]:

$$
\begin{aligned}
& f_{A}(a)=a \int_{0}^{\infty} \lambda J_{0}(a \lambda) \Lambda(\lambda) d \lambda,^{2} \\
& f_{R}(r)=r \int_{0}^{\infty} \lambda J_{0}(r \lambda) \exp \left(-\frac{\lambda^{2}}{2}\right) \Lambda(\lambda) d \lambda,
\end{aligned}
$$

where $J_{0}($.$) is the zero order Bessel function and:$

$$
\Lambda(\lambda)=E_{A_{1} \ldots A_{N}}\left[\prod_{i=1}^{N} J_{0}\left(A_{i} \lambda\right)\right]
$$

The PDF given in (9) completely characterizes the statistical behavior of the envelope in our general multipath fading channel model. However, its integral form is not convenient for analytic calculations, which are essential for mathematical analysis of communication systems in multipath fading channels. In this paper, we focus on Laguerre and power series to represent $f_{R}(r)$, which are more suitable for analytic studies. A review of different representations for $f_{R}(r)$ is provided in [3].

\section{EXPANSION of $f_{R}(r)$ In Terms of LAguerRe AND POWER SeRIES}

According to (7), it can be seen that the conditional PDF $f_{R \mid A}(r \mid a)$ is a Rice PDF [5]:

$$
f_{R \mid A}(r \mid a)=r \exp \left(-\frac{r^{2}+a^{2}}{2}\right) I_{0}(a r)
$$

where $I_{0}($.$) is the zero order modified Bessel function. After averaging with respect to A$ we obtain:

$$
f_{R}(r)=E_{A}\left[f_{R \mid A}(r \mid a)\right]=r \exp \left(-\frac{r^{2}}{2}\right) E_{A}\left[\exp \left(-\frac{A^{2}}{2}\right) I_{0}(A r)\right]
$$

${ }^{2}$ A Laguerre series for the PDF of $A$ is derived in [4]. For the case where the amplitudes $A_{i}$ 's are constant, one can use the recursive solution provided in [6]. 
The following generating function for Laguerre polynomials can be found in [7]:

$\exp (\sigma) J_{0}(2 \sqrt{\tau \sigma})=\sum_{n=0}^{\infty} \frac{L_{n}(\tau) \sigma^{n}}{n !}$

where $L_{n}($.$) is the Laguerre polynomial of order n$. For $\tau=-\beta r^{2} / 4$ and $\sigma=A^{2} / \beta$, (13) changes to:

$I_{0}(A r)=\exp \left(-\frac{A^{2}}{\beta}\right) \sum_{n=0}^{\infty} \frac{1}{n !} L_{n}\left(-\frac{\beta r^{2}}{4}\right)\left(\frac{A^{2}}{\beta}\right)^{n}, \quad \beta \neq 0$,

while for $\tau=A^{2} / \beta$ and $\sigma=-\beta r^{2} / 4$, (13) modifies to:

$I_{0}(A r)=\exp \left(\frac{\beta r^{2}}{4}\right) \sum_{n=0}^{\infty} \frac{1}{n !} L_{n}\left(\frac{A^{2}}{\beta}\right)\left(-\frac{\beta r^{2}}{4}\right)^{n}, \quad \beta \neq \pm \infty$

Equations (14) and (15) may be considered as parametric expansions for $I_{0}(A r)$ where the parameter $\beta$ is a real number, non-zero for (14) and finite in (15). For $\beta= \pm \infty$ in (14) and $\beta=0$ in (15), we obtain the Maclaurin series of $I_{0}($.$) :$

$I_{0}(A r)=\sum_{n=0}^{\infty} \frac{1}{(n !)^{2}}\left(\frac{A r}{2}\right)^{2 n}$

In order to proceed further, we need to define the following function:

$h_{n}(z)=E_{A}\left[\exp \left(-z A^{2}\right) A^{2 n}\right]$

where $z$ is an arbitrary real number and the PDF of $A$ is given in (8). This function will be discussed in detail in Section IV.

By replacing $I_{0}(A r)$ in (12) with (14) and (15), we obtain the Laguerre and the power series for $f_{R}(r)$, respectively:

$f_{R}(r)=\sum_{n=0}^{\infty} w_{n}(\beta) g_{n}(\beta, r), \quad \beta \neq 0$,

$f_{R}(r)=\sum_{n=0}^{\infty} v_{n}(\beta) d_{n}(\beta, r), \quad \beta \neq \pm \infty$,

in which:

$w_{n}(\beta)=\frac{1}{n ! \beta^{n}} h_{n}\left(\frac{1}{2}+\frac{1}{\beta}\right)$,

$g_{n}(\beta, r)=r \exp \left(-\frac{r^{2}}{2}\right) L_{n}\left(-\frac{\beta r^{2}}{4}\right)$, 
$v_{n}(\beta)=\frac{1}{n !} E_{A}\left[\exp \left(-\frac{A^{2}}{2}\right) L_{n}\left(\frac{A^{2}}{\beta}\right)\right]$,

$d_{n}(\beta, r)=r \exp \left(-\left(\frac{1}{2}-\frac{\beta}{4}\right) r^{2}\right)\left(-\frac{\beta r^{2}}{4}\right)^{n}$

In order to calculate the coefficient $w_{n}(\beta)$ in (18), only $h_{n}(1 / 2+1 / \beta)$ is required. However, for computing the coefficient $v_{n}(\beta)$ in (19), we need to determine the values of $h_{0}(1 / 2), \ldots, h_{n}(1 / 2)$, since according to the polynomial representation for $L_{n}($.$) [7]:$

$L_{n}(z)=\sum_{k=0}^{n} \frac{(-1)^{k} n !}{(n-k) !(k !)^{2}} z^{k}$

equation (22) can be written as:

$v_{n}(\beta)=\sum_{k=0}^{n} \frac{(-1)^{k}}{(n-k) !(k !)^{2} \beta^{k}} h_{k}\left(\frac{1}{2}\right)$.

Note that our starting point is the conditional Rice PDF in (11) and replacement of $I_{0}($.$) in (12)$ with an infinite series. The advantage of this approach, in comparison with expanding $f_{R}(r)$ directly in terms of an infinite series, is the fact that it provides a non-Rayleigh but perturbed-Rayleigh PDF. In other words, we usually expect a Rayleigh PDF for $f_{R}(r)$ in a multipath fading channel. In the presence of several strong multipath components which violate the CLT, $f_{R}(r)$ deviates from Rayleigh PDF. The amount of this deviation can be quantified in terms of the statistical properties of the amplitudes of these CLT-violating multipath components, if we use the conditional-Rice-PDF approach. This will be further discussed in Section IX.

\section{CLOSED FORM FORMULAS FOR $h_{n}(\cdot)$}

Based on the definition of $h_{n}(z)$ in (17) we have:

$h_{n}(z)=(-1)^{n} \frac{d^{n}}{d z^{n}} h_{0}(z)$

in which:

$h_{0}(z)=E_{A}\left[\exp \left(-z A^{2}\right)\right]$

Now we consider two cases separately. In the first case we assume that $f_{A}(a)$ is nonzero over the nonnegative real line. This assumption yields a simple solution for $h_{n}($.$) . In the second case, which is$ 
more general and also more realistic, $f_{A}(a)$ is assumed to be nonzero over an arbitrary finite interval. As we expect, the expressions derived for $h_{n}($.$) are more complicated in this case.$

A. $0 \leq A<\infty$

In this case we calculate the expectation in (17) directly. Equation (17) can be written as:

$h_{n}(z)=\int_{0}^{\infty} \exp \left(-z a^{2}\right) a^{2 n} f_{A}(a) d a$.

Based on the equation for $f_{A}(a)$ in (8) we obtain:

$h_{n}(z)=\int_{0}^{\infty} \exp \left(-z a^{2}\right) a^{2 n+1} \int_{0}^{\infty} \lambda J_{0}(a \lambda) \Lambda(\lambda) d \lambda d a$.

By the Fubini theorem [14], we can change the order of integration in (29). Based on the following relation [15]:

$\int_{0}^{\infty} a^{2 n+1} \exp \left(-z a^{2}\right) J_{0}(\lambda a) d a=\frac{n !}{2 z^{n+1}} \exp \left(-\frac{\lambda^{2}}{4 z}\right) L_{n}\left(\frac{\lambda^{2}}{4 z}\right), \quad z>0$,

it can be shown that:

$h_{n}(z)=\frac{n !}{z^{n}} \int_{0}^{\infty} \exp (-\xi) L_{n}(\xi) \Lambda(\sqrt{4 z \xi}) d \xi, \quad z>0$

Since $L_{n}($.$) is a polynomial given in (24), (31) can be rewritten as:$

$h_{n}(z)=\frac{(n !)^{2}}{z^{n}} \sum_{k=0}^{n} \frac{(-1)^{k}}{(n-k) !(k !)^{2}} h_{n, k}(z), \quad z>0$,

where:

$h_{n, k}(z)=\int_{0}^{\infty} \exp (-\xi) \xi^{k} \Lambda(\sqrt{4 z \xi}) d \xi, \quad z>0$

B. $A_{\min } \leq A \leq A_{\max }$

To calculate $h_{n}(z)$ for this case, first we derive a closed-form expression for $h_{0}(z)$ in (27) and then calculate the $n$th derivative in (26). By the straightforward extension of a result in [13], $h_{0}(z)$ can be expressed in terms of the hypergeometric series:

$h_{0}(z)=E_{A_{1} \ldots A_{N}}\left[\psi\left(N ; z A_{1}^{2}, \ldots, z A_{N}^{2}\right)\right], \quad z \geq 0$,

in which the hypergeometric series $\psi$ is defined as [13]: 
$\psi\left(N ; \xi_{1}, \ldots, \xi_{N}\right)=\sum_{m_{1}=0}^{\infty} \ldots \sum_{m_{N}=0}^{\infty}\left(m_{1}+\ldots+m_{N}\right) ! \prod_{k=1}^{N} \frac{\left(-\xi_{k}\right)^{m_{k}}}{\left(m_{k} !\right)^{2}}$

So:

$h_{0}(z)=\sum_{m_{1}=0}^{\infty} \ldots \sum_{m_{N}=0}^{\infty} \frac{\left(m_{1}+\ldots+m_{N}\right) !}{\prod_{k=1}^{N}\left(m_{k} !\right)^{2}} E_{A_{1} \ldots A_{N}}\left[\prod_{k=1}^{N} A_{k}^{2 m_{k}}\right](-z)^{m_{1}+\ldots+m_{N}}, \quad z \geq 0$.

Assuming that the multiple series in (36) converges, successive differentiation yields:

$h_{n}(z)=(-1)^{n} \sum_{m_{1}=0}^{\infty} \ldots \sum_{m_{N}=0}^{\infty} \frac{\left(m_{1}+\ldots+m_{N}\right) !}{\prod_{k=1}^{N}\left(m_{k} !\right)^{2}} E_{A_{1} \ldots A_{N}}\left[\prod_{k=1}^{N} A_{k}^{2 m_{k}}\right] \frac{d^{n}}{d z^{n}}(-z)^{m_{1}+\ldots+m_{N}}, \quad z \geq 0$

Equation (37) is useful for mathematical analysis of bit error probability in fading channels with arbitrary number of paths [21]. However, in numerical calculations, (37) is of limited use due to its multiple summation, specially when $N$ is large. Hence, in what follows we derive another formula for $h_{n}(z)$.

Apparently, direct calculation of the expectation in (17) is not possible, because in contrast with (30), there seems to be no useful closed-form solution for the following integral in terms of known mathematical functions:

$\int_{A_{\min }}^{A_{\max }} a^{2 n+1} \exp \left(-z a^{2}\right) J_{0}(\lambda a) d a$

Nevertheless, substitution of $\exp \left(-z a^{2}\right) J_{0}(\lambda a)$ with its associated Maclaurin series may yield useful results, because powers of $a$ can be easily integrated from $A_{\min }$ to $A_{\max }$. In [20] it is shown that:

$h_{n}(z)=\sum_{k=0}^{\infty} \frac{A_{\max }^{2 n+2 k+2}-A_{\min }^{2 n+2 k+2}}{2 n+2 k+2} \frac{(-z)^{k}}{k !} \int_{0}^{\infty} \lambda L_{k}\left(-\frac{\lambda^{2}}{4 z}\right) \Lambda(\lambda) d \lambda$.

Depending on the behavior of $\Lambda(\lambda)$, there might be cases where some of the integrals in (39) diverge, specially for large values of $k$. In situations where neither (37) nor (39) work, $h_{n}(z)$ has to be computed by numerical calculation of the expectation in (17), which is a double-fold integral:

$h_{n}(z)=\int_{A_{\min }}^{A_{\max }} \int_{0}^{\infty} \exp \left(-z a^{2}\right) a^{2 n+1} \lambda J_{0}(a \lambda) \Lambda(\lambda) d \lambda d a$.

So far, two infinite series have been introduced in (18) and (19) for $f_{R}(r)$, and several methods have been discussed for calculating their coefficients in (20) and (22), which are functions of $h_{n}($.$) .$ The kernels of these two series, given in (21) and (23), are of the general form $r^{m} \exp \left(-\alpha r^{2}\right)$, where 
$m$ is a nonnegative integer and $\alpha$ is a positive real number. Such a function is very convenient for mathematical manipulations, specially analytic integration, which usually appears in average bit error rate calculations, performance evaluation of diversity systems, etc. over multipath fading channels. Now the important issue is the number of terms which should be considered in those infinite series, in order to achieve a given truncation error. Note that for a fixed number of terms, the truncation error depends on $r$. So, for different values of $r$, different number of terms are required to obtain the same truncation error over the entire range $0 \leq r<\infty$. To avoid this problem, we need a uniform truncation error formula, which does not depend upon $r$. In the subsequent sections, we derive uniform upper bounds on the truncation errors of both series. Based on these bounds we can easily determine the minimum number of terms that one should take into account, in order to obtain a specified truncation error over the entire range of $r$.

\section{UPPER BOUND ON THE TRUNCATION ERROR OF LAGUERRE SERIES}

If we consider $n_{\max }+1$ terms in (18), the following truncation error appears:

$E_{n_{\max }}=\sum_{n=n_{\max }+1}^{\infty} w_{n}(\beta) g_{n}(\beta, r), \quad \beta \neq 0$.

Based on the triangular inequality we have:

$\left|E_{n_{\max }}\right| \leq \sum_{n=n_{\max }+1}^{\infty}\left|w_{n}(\beta)\right|\left|g_{n}(\beta, r)\right|, \quad \beta \neq 0$.

If we define the two upper bounds $q_{n}(\beta)$ and $u_{n}(\beta)$ as:

$q_{n}(\beta) \geq\left|w_{n}(\beta)\right|, \quad u_{n}(\beta)=\max _{r}\left|g_{n}(\beta, r)\right|$,

we obtain:

$\left|E_{n_{\max }}\right| \leq \sum_{n=n_{\max }+1}^{\infty} q_{n}(\beta) u_{n}(\beta)=\left|E_{n_{\max }}\right|_{\text {uub }}=e_{L}, \quad \beta \neq 0$,

in which the subscript $u u b$ stands for uniform upper bound. By uniform we mean that $\left|E_{n_{\max }}\right|_{u u b}$ (or simply, $\left.e_{L}\right)$ is an upper bound for $\left|E_{n_{\max }}\right|$ which is independent of $r$. The remainder of this section has been devoted to $u_{n}(\beta)$, while $q_{n}(\beta)$ will be discussed in Section VI.

The Laguerre polynomial $L_{n}(z)$ is positive and monotonically increasing for $z<0$, while for $z>0$ first it oscillates with $n$ zeros over the range $0<z<4 n$ (assuming $n$ is large) and then increases 
or decreases monotonically for $z>4 n$, provided that $n$ is even or odd, respectively [16]. For large $n$, Plancherel-Rotach type asymptotic formulas accurately describe the behavior of Laguerre polynomial:

$$
\begin{aligned}
& L_{n}(z) \approx \hat{L}_{n}(z)=\frac{1}{\sqrt{2 \pi n}}\left(\frac{z+\sqrt{z^{2}-4 n z}}{2 n}\right)^{2 n}\left(-\frac{z}{n}\right)^{-n} \exp \left(\frac{z-\sqrt{z^{2}-4 n z}}{2}\right)\left(\frac{z^{2}}{n^{2}}-\frac{4 z}{n}\right)^{-1 / 4}, \quad z>4 n, \\
& L_{n}(z) \approx \hat{L}_{n}(z)=\frac{(-1)^{n}}{\sqrt{\pi} n^{1 / 4}} z^{-1 / 4} \exp \left(\frac{z}{2}\right) \sin \left(\sqrt{n z}-n \pi+\frac{3 \pi}{4}\right), \quad 0<z<4 n, \\
& L_{n}(z) \approx \hat{L}_{n}(z)=\frac{1}{\sqrt{2 \pi n}}\left(\frac{-z+\sqrt{z^{2}-4 n z}}{2 n}\right)^{2 n+1}\left(-\frac{z}{n}\right)^{-n-1 / 2} \exp \left(\frac{z+\sqrt{z^{2}-4 n z}}{2}\right)\left(\frac{z^{2}}{n^{2}}-\frac{4 z}{n}\right)^{-1 / 4}, \quad z<0 .
\end{aligned}
$$

We have derived (45) and (46) from [17] after some simple manipulations, while (47) is given in [18] exactly. Note that besides the small differences between (45) and (47), which are due to the variations among the methods of approximation and representation used in [17] and [18], these two formulas can be obtained from each other by a simple reasoning [19].

According to the above properties of Laguerre polynomials and depending on $\beta,\left|g_{n}(\beta, r)\right|$ exhibits different behaviors versus $r$. Based on the unimodal shape of $r \exp \left(-r^{2} / 2\right),\left|g_{n}(\beta, r)\right|$ must have only one maximum for $\beta>0$, and $n+1$ maxima for $\beta<0$. Clearly, the first $n$ maxima fall into the region $0<r<\sqrt{-16 n / \beta}$, and the last one is out of this region, assuming $n$ is large. Using the SoninPolya theorem [17] [23], it is shown that if $n$ is large enough, then [20]:

1) for $\beta \leq-4$, the maxima of $\left|g_{n}(\beta, r)\right|$ constitute an increasing sequence, and,

2) for $-4<\beta<0$, the maxima of $\left|g_{n}(\beta, r)\right|$ form an increasing sequence for $r<\sqrt{2 /(\beta+4)}$, and a decreasing sequence for $r>\sqrt{2 /(\beta+4)}$.

Therefore, $u_{n}(\beta)$ takes different functional forms over the regions $\beta \leq-4,-4<\beta<0$, and $\beta>0$, as derived in [20]:

$$
\begin{aligned}
& u_{n}(\beta) \approx \hat{u}_{n}(\beta)=\sqrt{\frac{2 \beta n}{\beta+2}} \exp \left(-\frac{\beta n}{\beta+2}\right)\left|L_{n}\left(-\frac{\beta^{2} n}{2 \beta+4}\right)\right|, \quad \beta \leq-4 . \\
& u_{n}(\beta) \approx \hat{u}_{n}(\beta)=\sqrt{\frac{2}{\pi}\left(\frac{2}{e}\right)^{1 / 4}\left[\left(-\beta^{2}-4 \beta\right) n\right]^{-1 / 4}, \quad-4<\beta<0 .} \\
& u_{n}(\beta) \approx \hat{u}_{n}(\beta)=\sqrt{\frac{2 \beta n}{\beta+2}} \exp \left(-\frac{\beta n}{\beta+2}\right) L_{n}\left(-\frac{\beta^{2} n}{2 \beta+4}\right), \quad \beta>0 .
\end{aligned}
$$


Equations (48), (49), and (50) are asymptotic results, i.e. $\hat{u}_{n}(\beta) \rightarrow u_{n}(\beta)$ as $n \rightarrow \infty$. However, $\hat{u}_{n}(\beta)$ is very close to $u_{n}(\beta)$ even for $n$ as small as 4 , provided that $\beta$ is not very close to -4 or 0 . This can be easily verified, numerically [20].

\section{UPPER BOUND ON THE COEFFICIENTS OF LAGUERRE SERIES}

In order to obtain an upper bound on $h_{n}($.$) and then an appropriate expression for q_{n}(\beta)$ (upper bound on $\left|w_{n}(\beta)\right|$ for all possible joints distributions of $A_{i}$ 's), we assume that $A_{i}$ 's are bounded random variables, i.e.:

$$
A_{i} \leq A_{i, \max }, \quad i=1, \ldots, N
$$

Note that such an assumption is not restrictive, because $A_{i, \max }$ 's can be large. In general we have:

$$
A_{i, \min } \leq A_{i} \leq A_{i, \max }, \quad i=1, \ldots, N
$$

and for each $i, f_{A_{i}}\left(a_{i}\right)$ is zero outside the interval $\left[A_{i, \min }, A_{i, \max }\right]$. Hence:

$$
A_{\text {min }} \leq A \leq A_{\max }
$$

which means that $f_{A}(a)$ is nonzero only over a finite interval. It should be noted that $A_{\min }, A_{\max }, A_{i, \min }$ 's and $A_{i, \max }$ 's are all nonnegative constants. The following upper bound for $h_{n}($.) is derived in [20]:

$h_{n}(z)<K p_{n}(z)$

where:

$$
\begin{aligned}
& A_{\max }=\sum_{i=1}^{N} A_{i, \max }, \\
& K=\frac{A_{\max }}{\sqrt{\pi}} \int_{0}^{\infty} \sqrt{\lambda}|\Lambda(\lambda)| d \lambda, \\
& p_{n}(z)=\left\{\begin{array}{l}
\exp \left(-A_{\max }^{2} z\right) A_{\max }^{2 n+1 / 2}, \quad z<0 \\
A_{\max }^{2 n+1 / 2}, \quad z \geq 0
\end{array}\right.
\end{aligned}
$$

It should be noticed that depending on the behavior of $|\Lambda(\lambda)|$, there might be cases where the integral in (56) does not converge. In such cases, another upper bound should be found for $h_{n}($.$) . An$ example is discussed in the Appendix, where $N=2$ and $A_{1}$ and $A_{2}$ are two constants. This is the 
classic problem of two sine waves in Gaussian noise. For this special but important case, a finite upper bound is derived in [10] for $h_{n}($.$) . The details are given in the Appendix.$

The appropriate expression for $q_{n}(\beta)$ can be obtained by simply combining (20), (43), and (54):

$q_{n}(\beta)=\frac{K}{n !|\beta|^{n}} p_{n}\left(\frac{1}{2}+\frac{1}{\beta}\right), \quad \beta \neq 0$

Notice that the condition $\beta \neq 0$ in (58) comes from (18).

\section{CONVERGENCE OF $e_{L}$ AND OPTIMUM $\beta$ FOR THE LAGUERRE SERIES}

Based on the expressions derived for $q_{n}(\beta)$ and $u_{n}(\beta)$ in Sections VI and V, respectively, in this section we first investigate the convergence of $e_{L}$ in (44), an infinite series with positives terms, using the ratio test:

$\lim _{n \rightarrow \infty} \frac{q_{n+1}(\beta) u_{n+1}(\beta)}{q_{n}(\beta) u_{n}(\beta)}=Q(\beta) U(\beta), \quad \beta \neq 0$.

Here $Q(\beta)$ and $U(\beta)$ are defined as:

$Q(\beta)=\lim _{n \rightarrow \infty} \frac{q_{n+1}(\beta)}{q_{n}(\beta)}, \quad U(\beta)=\lim _{n \rightarrow \infty} \frac{u_{n+1}(\beta)}{u_{n}(\beta)}, \quad \beta \neq 0$

It is straightforward to verify that:

$Q(\beta)=\frac{A_{\max }^{2}}{|\beta|} \lim _{n \rightarrow \infty} \frac{1}{n}=0, \quad \beta \neq 0$.

For $U(\beta)$ we have three different cases, depending on the value of $\beta$. It is shown in [20] that:

$$
\begin{aligned}
& U(\beta)=-((\beta / 2)+1), \quad \beta \leq-4, \\
& U(\beta)=1, \quad-4<\beta<0, \\
& U(\beta)=(\beta / 2)+1, \quad \beta>0 .
\end{aligned}
$$

By putting together the expressions derived for $Q(\beta)$ and $U(\beta)$ in (61), (62), (63), and (64), we observe that:

$$
\lim _{n \rightarrow \infty} \frac{q_{n+1}(\beta) u_{n+1}(\beta)}{q_{n}(\beta) u_{n}(\beta)}=Q(\beta) U(\beta)=M_{L}(\beta) A_{\max }{ }^{2} \lim _{n \rightarrow \infty} \frac{1}{n}=0, \quad \beta \neq 0,
$$


where $M_{L}(\beta)$ is a finite positive constant, defined as:

$$
M_{L}(\beta)=\left\{\begin{array}{l}
(1 / 2)+(1 / \beta), \quad \beta \leq-4 \\
1 /|\beta|, \quad-4<\beta<0 \\
(1 / 2)+(1 / \beta), \quad \beta>0
\end{array} .\right.
$$

Since $Q(\beta) U(\beta)=0<1$, the infinite series $e_{L}$ in (44) converges for any $\beta \neq 0$ according to the ratio test. This also implies the convergence of Laguerre series in (18) for any $\beta \neq 0$, over the range $r \geq 0$, and for any joint distribution of $A_{i}$ 's.

In order to find an optimal value for $\beta$, we define the rate of convergence of $e_{L}$ as the optimality criterion. So, the optimal $\beta$ is the one which maximizes the rate of convergence of $e_{L}$, defined by $q_{n}(\beta) u_{n}(\beta) /\left[q_{n+1}(\beta) u_{n+1}(\beta)\right]$, where $n$ is a fixed large constant. This optimum value can be easily found by minimizing $M_{L}(\beta)$ in (66) over all $\beta$ 's:

$$
\inf _{\beta} M_{L}(\beta)= \begin{cases}1 / 4, & \beta \leq-4 \\ 1 / 4, & -4<\beta<0 \\ 1 / 2, & \beta>0\end{cases}
$$

According to (67), $\beta=-4$ turns out to be the optimum $\beta$, since it gives the minimum value of $M_{L}(\beta)$, i.e., maximizes the convergence rate of $e_{L}$ :

$$
\beta_{L, o p t}=-4 \Rightarrow M_{L}\left(\beta_{L, o p t}\right)=\frac{1}{4} \Rightarrow \frac{q_{n}\left(\beta_{L, o p t}\right) u_{n}\left(\beta_{L, o p t}\right)}{q_{n+1}\left(\beta_{L, o p t}\right) u_{n+1}\left(\beta_{L, o p t}\right)} \approx \frac{4 n}{A_{\max }^{2}}, \quad n \text { large }
$$

Such an optimum value for $\beta$ may be attributed to the orthogonality of Laguerre polynomials [11].

\section{COMParison OF THE OPTIMUM LAguerRe AND PoWER SERIES}

It has been proved in Section VII that $\beta=\beta_{L, \text { opt }}=-4$ optimizes the Laguerre series, whereas following the same approach, it can be shown that $\beta=\beta_{P, \text { opt }}=0$ optimizes the power series [20]. In order to compare these two series, we calculate their convergence rates according to (68):

$$
\frac{q_{n}(-4) u_{n}(-4)}{q_{n+1}(-4) u_{n+1}(-4)} \approx \frac{4 n}{A_{\max }^{2}}, \quad n \text { large }
$$

The convergence rate of the power series can be shown to be $2 n / A_{\max }{ }^{2}$, when $n$ is large [20]. As we can see, the convergence rate of the optimum power series is half of the optimum Laguerre series. 
Therefore, the optimum Laguerre series is superior from this point of view. By substituting (20) and (21) in (18) for $\beta=\beta_{L, \text { opt }}=-4$, the optimum Laguerre series can be written as:

$$
f_{R, o p t}(r)=r \exp \left(-\frac{r^{2}}{2}\right) \sum_{n=0}^{\infty} \frac{h_{n}(1 / 4)}{(-4)^{n} n !} L_{n}\left(r^{2}\right), \quad r \geq 0
$$

where $h_{n}(1 / 4)$ can be computed using any of the methods given in Section IV.

For $\beta=\beta_{L, \text { opt }}=-4$, substitution of $1.4 \hat{u}_{n}(-4)$ from (48) and $q_{n}(-4)$ from (58) into $e_{L}$ in (44) results in the following uniform upper bound on the truncation error of the optimum Laguerre series with $n_{\max }+1$ terms:

$e_{L, o p t}=2.8 K \sqrt{A_{\max }} \sum_{n=n_{\max }+1}^{\infty} \frac{\sqrt{n}}{n !}\left(\frac{A_{\max }}{2 e}\right)^{2 n}\left|L_{n}(4 n)\right|$,

where $A_{\max }$ and $K$ are given in (55) and (56), respectively. The factor 1.4 is added because it guarantees that $1.4 \hat{u}_{n}(-4) \geq\left|g_{n}(-4, r)\right|$ for all $n$ and $r$. This can be easily verified by visual comparison of the plots of $1.4 \hat{u}_{n}(-4)$ and $\left|g_{n}(-4, r)\right|$.

In a given problem with specific values for $e_{L, o p t}$ and $A_{\max }$, we can find a rough estimate for $n_{\max }$. According to (69) and for large $n$, the terms of the series $e_{L, o p t}$ in (71) have a maximum in the vicinity of $n=n_{p}$, assuming $A_{\max }$ is not too small, say $A_{\max } \geq 3$ :

$n_{p}=\left[\frac{A_{\max }^{2}}{4}\right]$,

where [.] gives the integer part. Since this maximum can be very large, specially for large $A_{\max }, n_{\max }$ should be several times greater than $n_{p}$, to obtain a small value for $e_{L, o p t}$.

\section{Applications ANd a Numerical Case Study}

As discussed at the end of Section III, our approach results in a non-Rayleigh but perturbedRayleigh PDF for the signal envelope in multipath fading channels. This important characteristic can be better understood by rewriting (70) as:

$$
f_{R, o p t}(r)=h_{0}(1 / 4) r \exp \left(-\frac{r^{2}}{2}\right)+\sum_{n=1}^{\infty} \frac{h_{n}(1 / 4)}{4^{n} n !}(-1)^{n} r \exp \left(-\frac{r^{2}}{2}\right) L_{n}\left(r^{2}\right), \quad r \geq 0 \text {. }
$$

Notice that [15]: 


$$
\int_{0}^{\infty} r \exp \left(-\frac{r^{2}}{2}\right) L_{n}\left(r^{2}\right) d r=(-1)^{n}
$$

Moreover, since the area under $f_{R, o p t}(r)$ is unity, according to (70) we must have:

$$
\sum_{n=0}^{\infty} \frac{h_{n}(1 / 4)}{4^{n} n !}=1
$$

Hence, the equation in (73) represents the non-Rayleigh envelope PDF as a mixture (more precisely, convex combination) of the Rayleigh $\operatorname{PDF} r \exp \left(-r^{2} / 2\right)$ and the unit-area kernels $(-1)^{n} r \exp \left(-r^{2} / 2\right) L_{n}\left(r^{2}\right), n=1,2, \ldots$. Notice that these kernels are not PDF since they take negative values for some $r$. However, it may be useful to consider them as pseudo PDF's since they integrate to one. When there is no strong multipath component, i.e. $N=0$, we have $h_{0}(1 / 4)=1$ and $h_{1}(1 / 4)=h_{2}(1 / 4)=\ldots=0$, which in turn yield $f_{R, o p t}(r)=r \exp \left(-r^{2} / 2\right)$. In the presence of at least one strong multipath component, i.e. when $N \geq 1$, we get $0 \leq h_{0}(1 / 4)<1$ while $h_{n}(1 / 4)>0$ for at least one $n$ from the set $\{1,2, \ldots\}$.

As an example of the insightful role of the mixture representation in (73) for analytic calculations in non-Rayleigh multipath fading channels, one can look at the problem of average bit error rate (BER) calculation in such channels [3]. In [3] it is shown that the average BER of any modulation method in multipath fading channels can be decomposed into two separate parts: the first part represents the average BER due to a large number of weak multipath components (which generate Rayleigh fading), whereas the second part shows the contribution of the few strong multipath components to the average BER. This representation provides a better understanding of the performance of communication systems and techniques in those wireless multipath indoor and outdoor channels, where the number of dominant multipath components is limited. A more comprehensive analysis of BER in multipath channels with a limited number of strong components is provided in [21].

Now we consider a numerical example and use the optimum Laguerre series to calculate the envelope PDF. Consider a multipath fading channel where in addition to the large number of weak multipath components, there are four CLT-violating multipath components: $A_{1}=0.5, A_{2}=1, A_{3}=3.5$ and $A_{4}=5$. Suppose that for representing the envelope PDF, we want to truncate the optimum Laguerre series in (70) with $n_{\max }+1$ terms such that $e_{L, o p t} \leq 10^{-4}$. Notice that according to (55) and (10) we have, respectively, $A_{\max }=10$ and $\Lambda(\lambda)=J_{0}(0.5 \lambda) J_{0}(\lambda) J_{0}(3.5 \lambda) J_{0}(5 \lambda)$. Using (71), it is easy to verify that $n_{\max }=75$ is the smallest integer which satisfies $e_{L, o p t} \leq 10^{-4}$. The coefficients $h_{n}(1 / 4)$ for 
$n=0,1, \ldots, 75$ are computed using (40) and are listed in [22]. Note that in our example $A_{\min }=0$. Since for any $n$ we have $h_{n}(1 / 4) \geq 0$, according to (75) we obtain $h_{n}(1 / 4) \leq 4^{n} n$ !, which is a tight upper bound only for small $n$.

The truncated form of the optimum Laguerre series in (70) with 76 terms, given by:

$$
f_{R, \text { opt }}^{\text {truncated }}(r)=r \exp \left(-\frac{r^{2}}{2}\right) \sum_{n=0}^{75} \frac{h_{n}(1 / 4)}{(-4)^{n} n !} L_{n}\left(r^{2}\right), \quad r \geq 0
$$

is plotted in Fig. 1. Note that as expected, it is nonnegative for any $r$. The average power of the signal envelope in our example is given by $E\left[R^{2}\right]=40.5$. An equivalent Rayleigh PDF $(r / 20.25) \exp \left(-r^{2} / 40.5\right)$, which has the same average power, is plotted in Fig. 1 as a reference. Fig. 1 also includes an equivalent Rice PDF $r \exp \left(-\left(r^{2}+38.5\right) / 2\right) I_{0}(\sqrt{38.5} r)$, with the same average power of 40.5. Note that this PDF is the same as the one in (12), where the random amplitude $A$ is replaced by the constant amplitude $\sqrt{38.5}$. Comparison of the plots in Fig. 1 reveals the inadequacy of Rayleigh and Rice models for characterizing the envelope PDF in the considered multipath channel. The natural logarithm of the truncation error of the Laguerre series, $e_{L}$ in (44), is plotted in Fig. 2 versus the parameter $\beta$. We notice in Fig. 2 that at $\beta=-4$, the truncation error attains its minimum. This agrees with our theoretical results which suggested $\beta=-4$ as an optimal choice.

\section{Conclusion}

In this paper we have considered a general model for multipath fading channels where in addition to a large number of weak multipath components, there are several strong multipath components. The presence of few dominant multipath components is more profound in wideband systems where the number of scatterers corresponding to a given delay bin decreases as the bandwidth increases [25], or in systems with smart antenna arrays, in which the receiver amplifies only few multipath components coming from particular directions and rejects the rest [12].

When there is no strong component, the envelope PDF reduces to Rayleigh, while the presence of at least one strong component results in a non-Rayleigh PDF for the envelope. There are several multipath fading models such as Nakagami, Weibull, lognormal, etc., which are not effective in characterizing the non-Rayleigh envelope PDF in some propagation environments [26] [27]. The proposed model of this paper is general enough to cover many cases of interest. The envelope PDF in our channel model can be expanded in terms of two polynomial-based infinite series: a Laguerre series 
and a power series, which both are very convenient for mathematical calculations. We have shown that the Laguerre series is preferred to the power series, since for a fixed number of terms it provides a smaller truncation error. Furthermore, we have derived an equation by which one can easily determine the minimum number of terms in the Laguerre series, which yields a specified truncation error over the entire positive real line. For the application of the Laguerre series to system performance analysis and bit error rate calculations, the interested reader can refer to [3] and [21]. The results derived in this paper can be used in other contexts such as radar clutter or light scattering, where the basic mechanism of random signal fluctuations can be modeled by the sum of random vectors.

\section{APPENDIX \\ ENVELOPE PDF of Two Sine WAVEs in GAussian Noise}

There are many cases, such as two path propagation environments [24], where we encounter the sum of two sine waves in Gaussian noise (see [10] for more examples). A variety of techniques for calculating this envelope PDF are discussed in [8]-[11].

If the two sine waves have uniformly distributed phases over $[0,2 \pi)$, then the envelope PDF of the sum of two sine waves in Gaussian noise is given by (9), where $N=2, A_{1}=a_{1}$, and $A_{2}=a_{2}\left(a_{1}\right.$ and $a_{2}$ two positive constants):

$$
f_{R}(r)=r \int_{0}^{\infty} \lambda J_{0}(r \lambda) \exp \left(-\frac{\lambda^{2}}{2}\right) \Lambda(\lambda) d \lambda, \quad r \geq 0 .
$$

Based on (10), $\Lambda(\lambda)$ in the above formula is:

$$
\Lambda(\lambda)=J_{o}\left(a_{1} \lambda\right) J_{o}\left(a_{2} \lambda\right)
$$

When there is no noise, the envelope PDF of two sine waves can be written in closed form by solving the integral in (8) [9]:

$$
f_{A}(a)=\left\{\begin{array}{l}
2 a /\left(\pi \sqrt{\left(a^{2}-a_{\min }^{2}\right)\left(a_{\max }^{2}-a^{2}\right)}\right), \quad a \in\left[a_{\min }, a_{\max }\right], \\
0, \quad a \notin\left[a_{\min }, a_{\max }\right],
\end{array}\right.
$$

where:

$$
a_{\min }=a_{1}-a_{2} \geq 0, \quad a_{\max }=a_{1}+a_{2} .
$$

For $A_{\min }=a_{\min }$ and $A_{\max }=a_{\max }$, the integral in (56) does not have a finite value. Therefore, the upper bound for $h_{n}(z)$ in (54) cannot be used in this case. Clearly, the upper bound $q_{n}(\beta)$ given in (58) does 
not work as well. In what follows, we present an appropriate upper bound for $h_{n}(z)$ and the corresponding expression for $q_{n}(\beta)$.

In [20] the following expression is derived for $h_{n}(z)$ :

$$
h_{n}(z)=\frac{\exp \left(-a_{\min }{ }^{2} z\right) a_{\min }{ }^{2 n} n !}{\sqrt{\pi}} \sum_{k=0}^{n} \frac{\Gamma(k+1 / 2)}{(n-k) !(k !)^{2}}\left(\frac{4 a_{1} a_{2}}{a_{\text {min }}{ }^{2}}\right)^{k}{ }_{1} F_{1}\left(k+\frac{1}{2}, k+1,-4 a_{1} a_{2} z\right),
$$

where ${ }_{1} F_{1}(., .,$.$) is the confluent hypergeometric function [15]. By obtaining an upper bound for$ ${ }_{1} F_{1}(., . .$.$) and after some algebraic manipulations, the following upper bound for h_{n}(z)$ is derived [20]:

$h_{n}(z) \leq \exp \left(4 a_{1} a_{2}|z|-a_{\min }^{2} z\right) a_{\max }^{2 n}$.

Comparison of (82) with (54), the upper bound on $h_{n}(z)$ for the general case of arbitrary $N$ and correlated $A_{i}$ 's in Section VI, reveals that both upper bounds are exponential in $n$. So, here we expect to obtain an expression similar to the one given in Section VI for $q_{n}(\beta)$, the upper bound on the coefficients of the Laguerre series in (18). In fact, by combining (20), (43), and (82) we obtain:

$q_{n}(\beta)=\exp \left(4 a_{1} a_{2}\left|\frac{1}{2}+\frac{1}{\beta}\right|-a_{\min }^{2}\left(\frac{1}{2}+\frac{1}{\beta}\right)\right) \frac{1}{n !|\beta|^{n}} a_{\max }^{2 n}, \quad \beta \neq 0$,

which has the same functional relationship with $n$ as $q_{n}(\beta)$ in (58).

Based on the similarities between the functional form of $q_{n}(\beta)$ in this appendix for $N=2$ and the one in Section VI for an arbitrary $N$, all the results of Section VII and subsequent sections hold here. This means that for the envelope PDF of two sine waves in Gaussian noise, the optimum Laguerre series $(\beta=-4)$ is superior to the optimum power series $(\beta=0)$. The optimum Laguerre series is the same as (70) in Section VIII, with $h_{n}(z)$ given in (81).

Similar to (71) in Section VIII, with $\beta=\beta_{L, o p t}=-4$, substitution of $1.4 \hat{u}_{n}(-4)$ from (48) and $q_{n}(-4)$ from (83) into $e_{L}$ in (44) results in the following uniform upper bound on the truncation error of the optimum Laguerre series with $n_{\max }+1$ terms:

$e_{L, o p t}=2.8 \exp \left(a_{1} a_{2}-\frac{a_{\min }^{2}}{4}\right) \sum_{n=n_{\max }+1}^{\infty} \frac{\sqrt{n}}{n !}\left(\frac{a_{\max }}{2 e}\right)^{2 n}\left|L_{n}(4 n)\right|$,

where $a_{\min }$ and $a_{\max }$ are defined in (80). Similar to (71), the factor 1.4 is added as it ensures that $1.4 \hat{u}_{n}(-4) \geq\left|g_{n}(-4, r)\right|$ for all $n$ and $r$. For a given $a_{\max }$ and based on the same type of argument used in Section VIII, $n_{\max }$ should be chosen much larger than $n_{p}=\left[a_{\max }^{2} / 4\right]$, if $e_{L, o p t}$ is required to be small. 
For $N=2$ and $\beta=-2,-4$, Laguerre series equivalent to (18) are derived in [9] and [11], respectively, via approaches different from ours. Nevertheless, the power series and its truncation error are not considered in [9] and [11], as well as the truncation error of the Laguerre series.

\section{REFERENCES}

[1] A. Abdi and M. Kaveh, "Envelope PDF in multipath fading channels with random number of paths and nonuniform phase distributions,” in Wireless Personal Communications: Emerging Technologies for Enhanced Communications. W. H. Tranter, T. S. Rappaport, B. D. Woerner, and J. H. Reed, Eds., Boston, MA: Kluwer, 1999, pp. 275-282.

[2] A. Papoulis, Probability, Random Variables, and Stochastic Processes, 3rd ed., Singapore: McGraw-Hill, 1991.

[3] A. Abdi, “A general framework for the characterization of multipath fading," in Proc. Conf. Inform. Sci. Syst., Princeton University, Princeton, NJ, 2002, pp. 414-418.

[4] A. Abdi, H. Hashemi, and S. Nader-Esfahani, "On the PDF of the sum of random vectors," IEEE Trans. Commun., vol. 48, pp. 7-12, 2000.

[5] P. Beckmann, Probability in Communication Engineering. New York: Harcourt, Brace \& World, 1967.

[6] M. K. Simon, "On the probability density function of the squared envelope of a sum of random phase vectors.” IEEE Trans. Commun., vol. 33, pp. 993-996, 1985.

[7] W. Magnus, F. Oberhettinger, and R. P. Soni, Formulas and Theorems for the Special Functions of Mathematical Physics, 3rd ed., New York: Springer, 1966.

[8] C. W. Helstrom, "Distribution of the sum of two sine waves and Gaussian noise," IEEE Trans. Inform. Theory, vol. 38, pp. 186-191, 1992.

[9] R. Esposito and L. R. Wilson, "Statistical properties of two sine waves in Gaussian noise," IEEE Trans. Inform. Theory, vol. 19, pp. 176-183, 1973.

[10] A. Abdi and S. Nader-Esfahani, “An optimum Laguerre expansion for the envelope PDF of two sine waves in Gaussian noise,” in Proc. IEEE Southeastcon Conf., Tampa, FL, 1996, pp. 160-163.

[11] R. Price, “An orthonormal Laguerre expansion yielding Rice's envelope density function for two sine waves in noise,” IEEE Trans. Inform. Theory, vol. 34, pp. 1375-1382, 1988.

[12] G. D. Durgin, T. S. Rappaport, and D. A. de Wolf, "More complete probability density functions for fading in mobile communications,” in Proc. IEEE Vehic. Technol. Conf., Houston, TX, 1999, pp. 985-989.

[13] M. Nesenbergs, "Error probability for multipath fading-The "slow and flat" idealization," IEEE Trans. Commun. Technol., vol. 15, pp. 797-805, 1967.

[14] J. D. DePree and C. W. Swartz, Introduction to Real Analysis. New York: Wiley, 1988.

[15] I. S. Gradshteyn and I. M. Ryzhik, Table of Integrals, Series, and Products, corrected and enlarged ed., A. Jeffrey, Ed., New York: Academic, 1980.

[16] J. Spanier and K. B. Oldham, An Atlas of Functions. Washington: Hemisphere, 1987.

[17] G. Szego, Orthogonal Polynomials, 3rd ed., Providence, RI: Amer. Math. Soc., 1967.

[18] W. Van Assche, Asymptotics for Orthogonal Polynomials. Lecture Notes in Mathematics, vol. 1265, Berlin: Springer, 1987.

[19] W. Van Assche, private communication, 1997. 
[20] A. Abdi, "Sum of random vectors problem and its applications in communication engineering," M.S. Thesis, Dept. Elec. Comp. Eng., University of Tehran, Feb. 1996.

[21] R. K. Bandila and A. Abdi, "On the performance limits in fading channels with arbitrary number of multipath components,” in Proc. Conf. Inform. Sci. Syst., Princeton University, Princeton, NJ, 2004, pp. 1186-1189.

[22] R. K. Choppala, “Alternative approaches to multipath fading,” M.S. Project Report, Dept. Elec. Comp. Eng., New Jersey Institute of Technology, Newark, NJ, Aug. 2003.

[23] A. F. Nikiforov and V. B. Uvarov, Special Functions of Mathematical Physics: A Unified Introduction with Applications. Basel, Germany: Birkhauser, 1988.

[24] T. Kurner, D. J. Cichon, and W. Wiesbeck, "Concepts and results for 3D digital terrain-based wave propagation models: An overview,” IEEE J. Select. Areas Commun., vol. 11, pp. 1002-1012, 1993.

[25] A. S. Molisch, "Mobile radio channels," in Wideband Wireless Digital Communications. A. F. Molisch, Ed., Upper Saddle River, NJ: Prentice Hall PTR, 2001, pp. 20-48.

[26] T. Kurner, D. J. Cichon, and W. Wiesbeck, "Evaluation and verification of the VHF/UHF propagation channel based on a 3-D-wave propagation model,” IEEE Trans. Antennas Propagat., vol. 44, pp. 393-404, 1996.

[27] M. Lebherz, W. Wiesbeck, and W. Krank, “A versatile wave propagation model for the VHF/UHF range considering three-dimensional terrain,” IEEE Trans. Antennas Propagat., vol. 40, pp. 1121-1131, 1992.

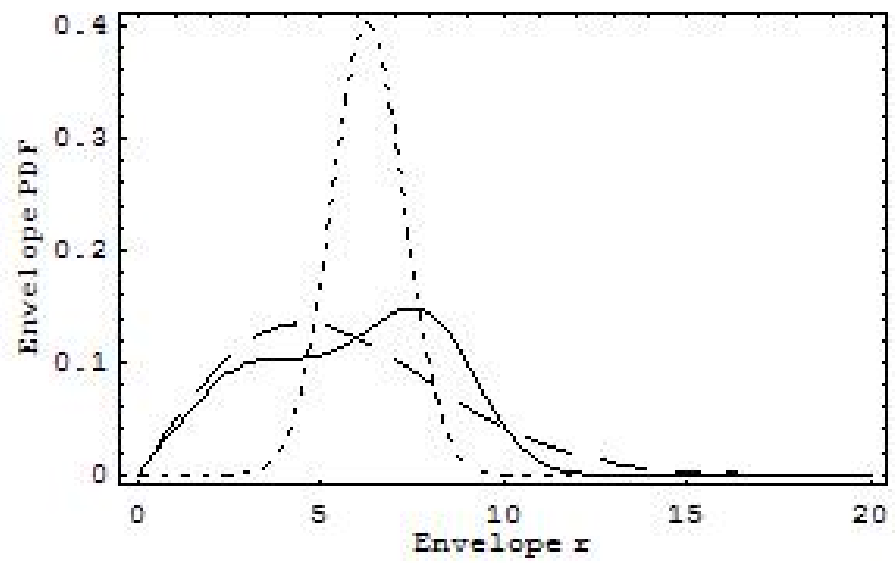

Fig. 1. The envelope PDF in a multipath fading channel with few strong multipath components: True PDF calculated using the proposed Laguerre series (solid), equivalent Rayleigh PDF (dashed), equivalent Rice PDF (dotted).

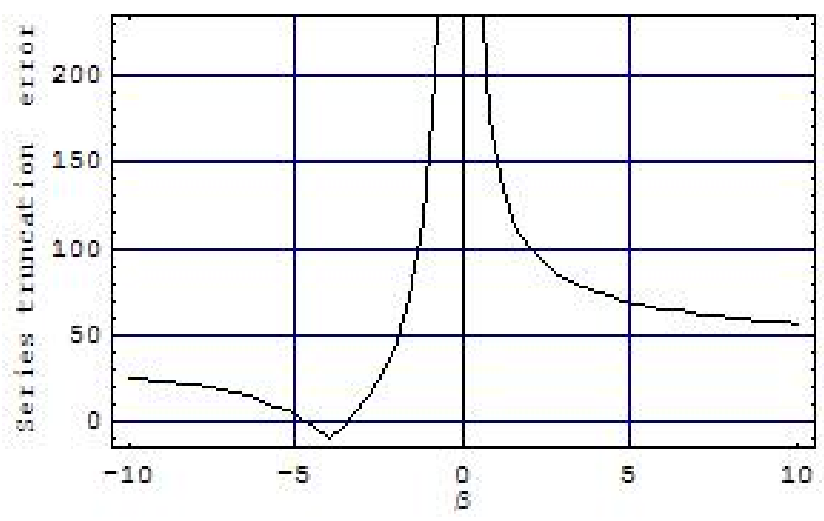

Fig. 2. Truncation error of the Laguerre series versus the parameter $\beta$. 


\section{List of Figure Captions:}

Fig. 1. The envelope PDF in a multipath fading channel with few strong multipath components: True PDF calculated using the proposed Laguerre series (solid), equivalent Rayleigh PDF (dashed), equivalent Rice PDF (dotted).

Fig. 2. Truncation error of the Laguerre series versus the parameter $\beta$.

\section{Author's Biography:}

Ali Abdi (S’98, M’01, SM’06) received the Ph.D. degree in electrical engineering from the University of Minnesota, Minneapolis, in 2001. He joined the Department of Electrical and Computer Engineering of New Jersey Institute of Technology (NJIT), Newark, in 2001, where he is currently an Associate Professor. His current research interests include characterization and estimation of wireless channels, digital communication in underwater and terrestrial channels, blind modulation recognition, systems biology and molecular networks. Dr. Abdi was an Associate Editor for IEEE Transactions on Vehicular Technology from 2002 to 2007. He was also the co-chair of the Communication and Information Theory Track of the 2008 IEEE ICCCN (International Conference on Computer Communications and Networks). Dr. Abdi has received 2006 NJIT Excellence in Teaching Award, in the category of Excellence in Team, Interdepartmental, Multidisciplinary, or Non-Traditional Teaching. He has also received 2008 New Jersey Inventors Hall of Fame (NJIHoF) Innovators Award on Acoustic Communication, for his work on underwater acoustic communication. 